Potenziale und Hindernisse interdisziplinärer Forschung

\title{
Auf dem Weg zu einer integrierten Nanotechnologieforschung?
}

\author{
Die Forschung zu Nanotechnologie ist ausgesprochen inter- \\ und transdisziplinär angelegt. Damit steht dieser Bereich vor \\ neuen Herausforderungen, die es zu meistern gilt. Wie kann \\ das Potenzial dieser Technologie zum Wohle der Gesellschaft \\ genutzt werden? Von Christian Pohl und Manuela Rossini
}

\begin{abstract}
m Jahr 2006 veranstalteten die Akademien der Wissenschaften Schweiz (akademien-schweiz) eine Tagung zur Frage, was die Nano- von der Gentechnologie bezüglich gesellschaftlicher Akzeptanz lernen kann. Die Tagung ergab, dass, verglichen mit der Gentechnologie, die Entwicklung der Nanotechnologie weniger stark durch die Industrie angetrieben wird, und dass Risiko- und ELSI-Forschung früher einbezogen werden (1). Einige der Expert(inn)en erarbeiteten auf Basis der Tagungsergebnisse ein Thesenpapier der Akademien der Wissenschaften Schweiz, dessen Kernpunkte in Abbildung 1 dargestellt sind (2).
\end{abstract}

\section{Intensiver Dialog zwischen Forschungsbereichen}

Für das td-net der akademien-schweiz, welches das Projekt leitete, waren besonders These zwei und drei von Interesse. In einem nächsten Schritt erfassten wir den Bestand der Dialogaktivitäten und der ELSI-Forschung in der Schweiz. Im Dialog ist das Zentrum für Technolo-

Abbildung 1: Thesen zur Nanotechnologie

1. Im Bereich der Nanotechnologie braucht es Forschung, die von den Interessen der Privatwirtschaft unabhängig ist.

2. Im Bereich der Nanotechnologie braucht es eine integrierte Grundlagen-, Risiko- und ELSI-Forschung.

3. Der Austausch zwischen Wissenschaft und Gesellschaft muss von den Forschenden proaktiv und als Teil ihrer Grundaufgabe geführt werden.

Quelle: Thesen zur Nanotechnologie, Akademien der Wissenschaften Schweiz, 2008
giefolgen-Abschätzung mit Bürgerforen und Studien zu Risiken und Chancen der Nanotechnologie sehr aktiv. Wir konzentrierten unser Engagement deshalb auf die integrierte Grundlagen-, Risiko- und ELSI-Forschung. 2008 luden wir zur Diskussion des Potenzials einer integrierten Risiko- und ELSI-Forschung in der Nanomedizin ein. Forschende aus der medizinischen Risikoforschung und aus der sozial-, geistes- und kulturwissenschaftlichen ELSI-Forschung diskutierten drei Fragen. Erstens, welchen inhaltlichen Beitrag die Risiko- von den ELSI-Forschenden benötigen, zweitens, welchen inhaltlichen Beitrag die ELSI- von den Risikoforschenden benötigen sowie drittens welches die Hürden und Potenziale für diese Zusammenarbeit sind.

Die Diskussion der ersten beiden Fragen verlief schwerfällig, obwohl die Teilnehmenden vorbereitet waren. Daraus schließen wir, dass es für Risiko- und ELSI-Forschende ungewohnt ist, ihre Forschung als Teil einer kollektiven Nanoforschung zu verstehen und den eigenen Beitrag zu den Beiträgen anderer Fachbereiche in Beziehung zu setzen. Lebhaft diskutiert wurden Hürden und Potenziale. Als Hürden wurden das Fehlen einer starken ELSI-Forschungsgemeinschaft in der Schweiz und eine eingespielte Arbeitsteilung zwischen Medizin, Naturund Ingenieurwissenschaften als für die Forschung und Geistes-, Sozial- und Kulturwissenschaften als für die Ethik zuständig identifiziert. Potenzial wurde im Einbezug gesellschaftlicher Akteure, in der Entwicklung beispielhafter Projekte und in der gemeinsamen Identifikation von Forschungsfragen erkannt.

\section{Wollen Forscher wirklich kooperieren?}

Die Bestandesaufnahme zeigte, dass die Schweiz bezüglich Dialogaktivitäten den internationalen Vergleich nicht zu scheuen braucht, wohingegen die ELSIForschung vergleichsweise schwach etabliert und finanziert ist. Auf internationaler Ebene stellten wir eine Tendenz weg von Forschung fest, die naturwissenschaftliche, technische und medizinische Entwicklung neuer Technologien durch separate geistes- und sozialwissenschaftliche Untersuchungen begleitet, hin zu einer integrierten Forschung. Darin arbeiten ELSI- und Risiko-Forschende mit Forschenden aus naturwissenschaftlichen und technischen Disziplinen zusammen an den Problemstellungen und Forschungsfragen, welche die neuen Technologien für Lehre, Forschung und Gesellschaft aufwerfen. Dadurch gestalten alle beteiligten Forschenden die Technologien aktiv mit.

Obwohl es verschiedentlich Bekenntnisse zur integrierten Forschung gibt, bleibt dies in der Schweiz derzeit noch Zukunftsmusik. Ein Grund dafür ist, das nicht klar ist, in wieweit sie von den Beteiligten selbst tatsächlich gewünscht wird, denn Nanotechnologie-Forschende sehen ihren Gestaltungsfreiraum eingeschränkt während ELSI-Forschende gar nicht unbedingt mitgestalten wollen.

\section{Anmerkungen}

(1) ELSI steht für Ethical, Legal and Social Implications

(2) Das Thesenpapier und der Bericht zur integrierten Forschung finden sie im Internet unter: http://www.transdisciplinarity.ch/d/Publications

I AUTOREN + KONTAKT

Dr. Christian Pohl ist Co-Leiter und Dr. Manuela Rossini ist wissenschaftliche Projektleiterin am td-net, dem Network for Transdisciplinary Research der Akademien der Wissenschaften Schweiz.

td-net, Schwarztorstrasse 9, 3007 Bern, Schweiz. Tel +41 3131040 94; Fax +41 313121678 E-Mail: td-net@scnat.ch 
(c) 20I0 Authors; licensee IÖW and oekom verlag. This is an article distributed under the terms of the Creative Commons Attribution Non-Commercial No Derivates License (http://creativecommons.org/licenses/by-nc-nd/3.o/), which permits unrestricted use, distribution, and reproduction in any medium, provided the original work is properly cited. 